\title{
P03-4-29 Poster session
}

\section{Airway nociceptor neurons detect allergens after sensitization}

\section{Sebastien Talbot}

\section{Pharmacology and Physiology, Universite de Montreal, Canada}

Both the immune and nervous systems detect danger and initiate coordinated responses to threats in a manner that includes bidirectional communication and functional integration. Lung nociceptor neurons, for example, contribute to a feed-forward pro-inflammatory loop with immune cells during type 2 allergic inflammation. However, the cellular mechanisms by which sensory neurons are first activated after an allergen challenge to initiate this loop are unknown. Here, we show that an allergen challenge in OVA-sensitized mice rapidly triggers action potential firing in vagal nociceptors, well before immune cells are recruited to the lungs, while silencing these neurons prior to the first allergen challenge reduces immune cell infiltration. Nodose ganglion neurons express FceR1 whose level increases in sensitized mice. These neurons when obtained from sensitized mice respond in vitro directly to allergen complexed with $\operatorname{IgE}$ with decreased resting membrane potentials, action potential firing and a TRPC3 channel dependent calcium influx. FceR1a genetic ablation or pharmacological blockade prevents this activation of nociceptors by IgE-OVA. We conclude that in sensitized animals, allergen sensing by airway nociceptors through FceR1 triggers neuropeptide release to contribute to immune cell influx. Targeting FceR1-nociceptor interactions may represent a novel strategy, therefore, to prevent initiation of allergic airway inflammation. 